\title{
X-ray analysis of stacking faults in ytterbium
}

KALYANI VIJAYAN

Materials Science Division, National Aeronautical Laboratory, Bangalore 560 077. India

At room temperature $(\approx 300 \mathrm{~K})$, ytterbium has a predominantly fcc structure $[1,2]$. However, a small amount of the hcp structure corresponding to the low- as well as the high-temperature phases [3-6] is also known to coexist $[7,8]$ with the room-temperature fcc structure. We have earlier reported [8] that mechanical deformation of $99.2 \%$ pure ytterbium specimens at room temperature, induces a partial structural transformation of the type $\mathrm{fcc} \rightarrow \mathrm{hcp}$, leading to an enhancement of the $\mathrm{hcp}$ component in the deformed material. It has also been found [9] that surfaces of ytterbium specimens subjected to polishing with emery or to filing also exhibit similar enhancement of the $\mathrm{h} \mathrm{c} \mathrm{p}$ component. These observations suggest that in $99.2 \%$ pure ytterbium, the $\mathrm{fcc} \rightarrow \mathrm{hcp}$ transformation which readily accompanies different types of mechanical treatments, is probably associated with the stacking faults in the material.

It has also been observed that the unit cell dimensions [4] of the fcc and the h c p phases of ytterbium match in the following manner: $c_{\mathrm{hcp}} \approx 2 / 3$ body diagonal of the $\mathrm{fcc}$ cell, i.e.

$$
0.638 \approx 2 / 3(0.949)=0.633 \mathrm{~nm}
$$

and $a_{\mathrm{hcp}} \approx 1 / 2$ face diagonal of the fcc cell, i.e.

$$
0.388 \approx 1 / 2(0.775)=0.387 \mathrm{~nm}
$$

These relationships indicate that the $c$ and $a$ axial directions of the $\mathrm{h} c \mathrm{p}$ cell are likely to be parallel to the [1 111$]$ and the [1 10 ] directions of the f c c cell.

The equivalence represented in Equation 1 can be derived by using the concept of stacking faults. It is well known that in the f c c structure, the stacking sequence along the three-fold axis is represented as ABCABC - - - . When the stacking sequence is ideal, as above, the repeat distance between any two stacks of the same type, i.e. A to $\mathbf{A}, \mathbf{B}$ to $\mathbf{B}$ or $C$ to $\mathbf{C}$, is equal to the length of the body diagonal of the face centred cell. However, when there is a stacking fault of the type $\mathbf{A B} \downarrow \mathbf{A B C}---$, the repeat distance is reduced in that region. Here, the arrow represents a break in the sequence or a stacking fault. In the case of a single stacking fault, the repeat distance is reduced to twothirds its original value. Also, the single fault produces four layers, ABAB, of the h c p sequence. When deformation stacking faults of this type occur frequently, it may be expected that the $\mathrm{h} \mathrm{c} \mathrm{p}$ sequence derived from the f c c sequence, by virtue of the stacking faults, will be large enough to be detected by X-rays. With a view to examining the proposed correlation between stacking faults and the deformation-induced structural transformation, the stacking fault probability in 99.2\% pure ytterbium has been determined by X-ray diffraction methods and the results are presented in this letter.

Details of the impurities in the ytterbium samples have been described elsewhere [8]. Cold working of block ytterbium specimens was carried out by hand filing at room temperature. To prevent oxidation, the filing was carried out in a medium of kerosene oil. The filings were dried on filter paper. Some of the cold-worked filings were sealed under vacuum and were annealed at $225 \pm 2$ " C for $3 \mathrm{~h}$. X-ray patterns from the annealed and cold-worked filings were recorded on a Philips powder diffractometer using $\mathrm{CuK \alpha}$ radiation, proportional counter and a graphite monochromator in the diffracted beam. The profiles of the reflections (11 1), (200), (220) and (311) were recorded using a sample rotation speed of $1 / 8^{\circ} \mathrm{min}^{-1}$ and a chart speed of $20 \mathrm{~mm} \mathrm{~min}^{-1}$. In addition to the chart recording, point counts were also collected for the reflections ( 1111$)$ and (200). In the neighbourhood of the peak, the 28 interval was chosen as 0.01 " and in the background regions, it was varied from 0.02 to $0.04 "$. The counting time was also appropriately varied to ensure that even in the background regions of the profile, for each 28 value, the total number of counts was at least 10000 .

Values of the deformation fault probability, $\boldsymbol{a}$, and the twin fault probability, $\beta$, have been estimated from the observed peak shifts and the peak asymmetry, respectively. 28 values corresponding to the peak maximum, $2 \theta_{\max }$, have been determined from the chart-recorded profiles using the mid-point chord extrapolation method [10]. The centres of gravity (C.G.) of the point-counted profiles have been calculated by adopting the method of Ladell et al. [1 I].

The average value of $a$ has been estimated from the $2 \theta_{\max }$ values of the neighbouring pairs of reflections $(200)-(200)$ and (31 1)-(220), using the relations [12]

$$
\begin{aligned}
\Delta\left[\Delta\left(2 \theta^{\circ}\right)\right]= & \left(2 \theta_{200}^{\circ}-2 \theta \Gamma 11\right)^{\text {cold-worked }} \\
& -\left(2 \theta_{200}^{\circ}-2 \theta_{111}^{\circ}\right)^{\text {annealed }} \\
= & \frac{-90 \sqrt{3}}{\pi^{2}} \alpha\left(\frac{\tan \theta_{200}}{2}+\frac{\tan \theta_{111}}{4}\right) \\
\Delta\left[\Delta\left(2 \theta^{\circ}\right)\right]= & \frac{90 \sqrt{ } 3}{\pi^{2}} \alpha\left(\frac{\tan \theta_{220}}{4}+\frac{\tan \theta_{200}}{2}\right)
\end{aligned}
$$

and

$$
\Delta\left[\Delta\left(2 \theta^{\circ}\right)\right]=\frac{-902 \sqrt{3}}{\pi^{2}} \alpha\left(\frac{\tan \theta_{311}}{11}+\frac{\tan \theta_{220}}{4}\right)
$$

Table I lists the directions and magnitudes of the 
T A B LE I Peak shifts, deformation $(x)$ and twin fault $(\beta)$ probabilities in cold-worked ytterbium

\begin{tabular}{lccc}
\hline Reflections & $\Delta\left[\Delta\left(2 \theta^{\circ}\right)\right]$ & $\chi \times 10^{\prime}$ & $\beta \times 10^{3}$ \\
\hline$(200)-(1111)$ & -0.083 & 25.0 & 10.2 \\
$(220)-(200)$ & +0.073 & 18.3 & \\
$(3$ I 1 $)-(220)$ & -0.080 & 32.5 & \\
& & Av. 25.3 & \\
\hline
\end{tabular}

observed shifts $\Delta\left[\Delta\left(2 \theta^{\circ}\right)\right]$. The agreement between the observed directions of shift and those predicted by Paterson [13] confirm that the observed effects are due to stacking faults. The average value of a $\times 10^{3}=25$ indicates that in the cold-worked filings, on an average, every 40th (1 111 ) plane is faulted. This corresponds to an average separation of $40 d_{11},=12.7 \mathrm{~nm}$ between the faulted planes.

The twin fault probability, $\beta$, has been evaluated from the relation [14]

$$
\beta=\frac{\Delta . C . G .\left(2 \theta_{111}^{\circ}\right)-\Delta \cdot C \cdot G \cdot\left(2 \theta_{200}^{\circ}\right)}{11 \tan \theta_{111}+14 \cdot 6 \tan \theta_{200}}
$$

where $\Delta$. C.G. $\left(2 \theta_{h k l}^{\circ}\right)=\left(2 \theta_{\mathrm{C} . \mathrm{G}}^{\circ}-2 \theta_{\mathrm{max}}^{\circ}\right)_{h k l}$. The value of $\beta$ presented in Table I indicates that 1 in every 98 (1 11) plane is likely to be faulted as ABCACBA.

The accuracy of the calculation of $\beta$ is admittedly low because it has been assumed that the observed peak asymmetry is entirely due to twin faults. The contribution to peak asymmetry from segregation of impurities [15] cannot be ruled out, especially for the specimens which are only $99.2 \%$ pure. Hence, no further discussion of the $\beta$ value has been attempted in this letter.

The average, deformation probability, a, of ytterbium is found to be rather high and is comparable to that observed in a-brass [16]. Based on the inverse relationship between a and the stacking fault energy [17], it may be concluded in a quantitative manner, that the ytterbium samples are characterized by low stacking fault energy. We have earlier observed that in deformed ytterbium, recrystallization occurs at $\approx 400 \mathrm{~K}$ [18]. From the correlation between stacking fault energy and recrystallization given by Cotterill and Mould [19] it appears that the recrystallization in ytterbium is also due to its low stacking fault energy.

Based on the stacking fault probabilities, the matching of the unit cells of the $\mathrm{fcc}$ and the deformationinduced $\mathrm{hcp}$ phases and the recrystallization, it is concluded that the deformation-induced transformation in ytterbium is essentially a stacking fault effect.

\section{Acknowledgement}

The author thanks Dr A. K. Singh for the many useful suggestions and Dr T. G. Ramesh for kindly supplying the ytterbium samples.

\section{References}

1. F. H. SPEDDING, J. J. HANAK and A. H. DAANE, J. Less-Common Metals 3 (1963) 110.

2. A. JAYARAMAN, Phys. Rev. A135 (1964) 1056.

3. C. M. HURD and J. E. A. ALDERSON, Solid State Commun. 12 (1973) 375.

4. F. X. KAYSER, Phys. Rev. Lett. 25 (1970) 662.

5. S TANUMA, W. R. DATARS, H. DOI and A. DUNS. WORTH, Solid State Commun. 8 (1970) 1107.

6. M. V. VEDERNIKOV, A. T. BURKOV, V. G. DVUNITKIN and N. I, MOREVA, Phys. Lett. $\boldsymbol{A} 48$ (1974) 293.

7. E. BUCHER, P. H. SCHMIDT, A. JAYARAMAN and K. ANDRES, Phys. Rev. 2 (1970) 3911.

8. A. MANI and KALYANI VIJAYAN, Curr. Sci. 53 (1984) 28.

9. A. MANI and KALYANI VIJAYAN, unpublished.

10. J. A. BEARDEN, Phys. Rev. 43 (1933) 92.

11. J. I.ADELI. W. PARRISH and J. TAYLOR, Acta Crystallogr. 12 (1959) 253.

12. C. N. J. W A GNER, Acta Metall. 5 (1957) 427.

13. M. S. PAterson. J. Appl. Phys. 23 (1952) 805

14. J. B. COHEN and C. N. J. WAGNER, ibid. 33 (1962) 2073.

15. B. T. M. WILlis, Acta Crystallogr. 12 (1959) 683.

16. B. E. WARREN and E. P. WAREKOIS, Acla Metall. 3 (1955) 473.

17. L. F. VAS S A millet, J. Appl. Phys. 32 (1961) 778.

18. A. MANI and KALYANI and VIJAYAN, J. Mater. Sci. Lett. 6 (1987) 663.

19. P. COTTERILL and P. R. MOULD, "Recrystallization and grain growth in metals" (Surrey University Press, London, 1976).

Received 3 February

and accepted 20 April 1988 